Phys. Rev. D54, 10/1/96

hep-ph/9608259

\title{
Pion Helicity Structure And Its Consequence For The Hard Scattering Form Factor
}

\author{
S.W. Wang' and L.S. Kisslinger \\ Physics Department, Carnegie-Mellon University, Pittsburgh, PA 15213, USA
}

\begin{abstract}
In a study of the elastic pion form factor for large momentum transfers based on a modified perturbative QCD (PQCD) approach we have included helicity components that are customarily neglected. Along with the inclusion of transverse momentum, this gives a large suppression of the form factor from the prediction of the original hard scattering model based on PQCD in the $Q^{2}$ domain where experimental data are available. We conclude that nonperturbative contributions will dominate in this region.
\end{abstract}

PACS numbers: 13.40.G, 13.60, 12.38.B,C, 12.39

\footnotetext{
${ }^{1}$ Present address: Department of Physics, University of Maryland, College Park, MD 20742
} 
It has been suggested that the hard scattering model for the hadronic form factors [1] derived using perturbative QCD (PQCD) is applicable in the currently experimentally accessible regime of momentum transfer $\left(Q^{2} \sim\right.$ a few $\left.\mathrm{GeV}^{2}\right)$. Facing critical questions and doubts [2] about whether it is justified to use the quark distribution function proposed by Chernyak-Zhitnitsky (CZ) [3], which can increase the hard scattering form factor to improve consistency with experiment, Li and Sterman [4] recently proposed a scheme with Sudakov suppression included in an attempt to make the hard scattering formalism internally consistent. They started with a modified expression of hard scattering amplitude $T_{H}$ in which they had retained the transverse momentum in the gluon propagator. The application of the renormalization group to both the wave function and the hard scattering amplitude $T_{H}$ allowed them to pinpoint the dangerous soft end-point region and associate it with the transverse distance. They conclude that with the inclusion of the Sudakov correction the hard scattering form remains valid and [with a CZ quark distribution] is comparable to the experimental data for the $Q^{2}$ in as low as about $1 \mathrm{GeV}^{2}$ for the pion.

However, in our early work on the calculation of pion elastic form factor in the light-cone Bethe-Salpeter (BS) formalism [5], and in our recent work [6] in which a Sudakov form factor and other effects have been included, we consistently find a dominant soft contribution (i.e. form factor arising from the confining kernel as defined in [5, 6]) in the few $\mathrm{GeV}^{2}$ region. With the inclusion of transverse momentum in both the soft wave function and the onegluon exchange integral kernel there is strong suppression of hard scattering at a few $\mathrm{GeV}^{2}$. This effect has also been pointed out by Jacob and Kroll in their recent work[0], but they have dropped helicity components in the soft wave function which do not contribute in the conventional model.

With the inclusion of transverse momentum there are contributions to the hard scattering process which are not included in the standard [1] hard scattering formula. It is the objective of the present note to reanalyse the pure hard scattering pion form factor using the modified $T_{H}$ but without the exclusion of the transverse momentum or the unconventional helicity components in the soft wave function. We show that these effects produce important changes in the results of the hard scattering prediction in the region of momentum transfer where current experimental data are available. In this note we will be using two models for the soft wave function which give satisfactory descriptions for low $Q^{2}$ properties of the form factor.

Let us first review the light-cone wave functions [BS amplitudes] needed for the hard scattering form. The transformation of the single particle states between different representations can be formulated using their identity at zero momentum. For instance, the instant form of a single-particle state is related to the light-cone representation by the following equation 8 ,

$$
|\mathbf{p} \mu\rangle=\sum_{\bar{\mu}}|\mathbf{p} \bar{\mu}\rangle_{l . c .} D_{\bar{\mu} \mu}^{j}\left[R_{f c}(p)\right]
$$

where $R_{f c}(p)$ is called the Melosh rotation [9] and the subscript l.c denotes light-cone representation. It is easy to work out Eq. (1) for $j=1 / 2$, which is 8 


$$
|\mathbf{p} \uparrow(\downarrow)\rangle=\frac{\left(p^{+}+m\right)|\mathbf{p} \uparrow(\downarrow)\rangle_{l . c .} \mp p^{R(L)}|\mathbf{p} \downarrow(\uparrow)\rangle_{l . c .}}{\sqrt{2 p^{+}\left(p^{0}+m\right)}},
$$

where $p^{R(L)}=p_{x} \pm i p_{y}$ and $m$ is the quark mass.

This result, that a helicity state in an instant-form representation is a mixed helicity state in the light-cone representation, is hardly a surprise since a "rotationless boost" defined in the light-cone representation is not a pure boost any more when viewed in the instant form and vice versa. In other words, the transform from one representation to another inevitably causes a state to undergo a "rotation".

To construct a state for a pion in the light-cone form, one starts with a quark-antiquark pair being coupled to form a spin zero state in the pion rest frame, and then boosts it to an arbitrary momentum using Eq. (2). Using the standard light-cone notation of $\left(x, \mathbf{k}_{\perp}\right)$ for the longitudinal and transverse relative momentum, we tabulate the explicit form of the wave functions along with their Fourier transforms in the Table 1 and Table 2. One sees that there do exist four helicity components, namely

$$
\Psi=\left(\Psi_{\uparrow \uparrow}, \Psi_{\uparrow \downarrow}, \Psi_{\downarrow \uparrow}, \Psi_{\downarrow \downarrow}\right) .
$$

For simplicity of illustration, in the present work we consider two models of pion helicity functions. One is obtained by a naive extension from nonrelativistic spin form to the relativistic light-cone form

$$
\frac{1}{\sqrt{2}} \chi^{\dagger}(1) i \sigma_{2} \chi(2) \longrightarrow \chi_{\pi}=\frac{1}{\sqrt{2}} \bar{u}(1) \gamma_{5} v(2)
$$

Another is suggested by Dziembowski[10]

$$
\chi_{\pi}=\bar{u}(1)\left(p_{\mu} \gamma^{\mu}+m_{\pi}\right) \gamma_{5} v(2) .
$$

The light-cone spinors $u$ and $v$ have the Melosh rotation built into them. The helicity function given by Eq. (5) is a four-component generalization of the one obtained by a Melosh rotation of the wave function given on the left-hand side of Eq. (4), apart from an overall factor. For the confinement wave functions in momentum space we have adapted the Brodsky-Huang-Lepage's oscillator prescription[1].

The modified $T_{H}$ is obtained by retaining the transverse momentum in the gluon propagator[, which gives

$$
T_{H}\left(x, y, \mathbf{k}_{\perp}, \ell_{\perp}, Q, \mu\right)=\frac{4 g^{2}(\mu) C_{F}}{(1-x)(1-y) Q^{2}+\left(\mathbf{k}_{\perp}-\ell_{\perp}\right)^{2}} .
$$

\footnotetext{
${ }^{2}$ The transverse momentum in the quark propagator has been neglected for the simplicity of analysis. This is sufficient for the purpose of our present work for it is known [4] that such effect gives about $15 \%$ correction for the form factor at $2 \mathrm{GeV}^{2}$, about an order of magnitude smaller than the effects we find for additional helicity components. Moreover, these additional corrections would further suppress the PQCD contribution.
} 
Li-Sterman[ []] introduced a Fourier transform in transverse momentum, leading to the modified hard scattering pion form factor:

$$
F_{\pi}\left(Q^{2}\right)=\int \frac{d x d y}{\left(16 \pi^{3}\right)^{2}} \int \frac{d^{2} b}{(2 \pi)^{2}} \hat{\Psi}^{*}(y, \mathbf{b}) \hat{T}_{H}(x, y, \mathbf{b}, Q, t) \hat{\Psi}(x, \mathbf{b}) \exp (-S(x, y, b, Q, t)),
$$

where the Fourier transform of $T_{H}\left(x, y, \mathbf{k}_{\perp}, \ell_{\perp}, Q, \mu\right)$ is

$$
\hat{T}_{H}(x, y, \mathbf{b}, Q, t)=32 \pi^{2} C_{f} \alpha_{s}(t) K_{0}(\sqrt{(1-x)(1-y)} Q b),
$$

with $K_{0}(z)$ being the modified Bessel function, and

$$
t=\max (\sqrt{(1-x)(1-y)} Q, 1 / b)
$$

The Sudakov factor $S(x, y, b, Q, t)$ is given in detail in [1]. In deriving Eq. (6) and Eq. (77), it has been assumed that only those conventional helicity components, namely $h_{1}+h_{2}=0$, are relevant. In order to include the $h_{1}+h_{2}= \pm 1$ components in addition, one needs to find out their corresponding $T_{H}$.

Taking into account the symmetries of our model wave functions, especially the fact that $\Psi_{\uparrow \uparrow}^{*}\left(x, \mathbf{k}_{\perp}\right)=\Psi_{\downarrow \downarrow}\left(x, \mathbf{k}_{\perp}\right)$, and under the assumption that $T_{H}$ depends only on $\mathbf{k}=\mathbf{k}_{\perp}-\ell_{\perp}$, it is straightforward to show that

$$
T_{H}(x, y, \mathbf{k}, Q, \mu)^{\uparrow \downarrow+\downarrow \uparrow}=-T_{H}(x, y, \mathbf{k}, Q, \mu)^{\uparrow \uparrow+\downarrow \downarrow} .
$$

One will see later that the minus sign in $T_{H}$ associated with $\uparrow \uparrow+\downarrow \downarrow$ components here has the crucial consequence that the unconventional helicity components will indeed generate a suppression on the pion form factor.

Table 1: The naive wave function $\Psi\left(x, \mathbf{k}_{\perp}\right)$ and its $\mathrm{FT} \hat{\Psi}(x, \mathbf{b})$

\begin{tabular}{|c|c|c|}
\hline$\lambda_{1} \lambda_{2}$ & $\Psi_{\lambda_{1} \lambda_{2}}\left(x, \mathbf{k}_{\perp}\right)$ & $\hat{\Psi}_{\lambda_{1} \lambda_{2}}(x, \mathbf{b})$ \\
\hline$\uparrow \uparrow$ & $-k^{L} \hat{\phi}\left(x, k_{\perp}\right)$ & $i b^{L} \xi(x, b) g(x) / 2$ \\
\hline$\uparrow \downarrow$ & $m \hat{\phi}\left(x, k_{\perp}\right)$ & $m \xi(x, b)$ \\
\hline$\downarrow \uparrow$ & $-m \hat{\phi}\left(x, k_{\perp}\right)$ & $-m \xi(x, b)$ \\
\hline$\downarrow \downarrow$ & $-k^{R} \hat{\phi}\left(x, k_{\perp}\right)$ & $i b^{R} \xi(x, b) g(x) / 2$ \\
\hline
\end{tabular}

The complete wave functions and their corresponding Fourier transforms (FT) are given explicitly in the Table 1 and Table 2, where the FT is defined as

$$
\Psi\left(x, \mathbf{k}_{\perp}\right)=\int \frac{d^{2} b}{(2 \pi)^{2}} \hat{\Psi}(x, \mathbf{b}) e^{-i \mathbf{b} \cdot \mathbf{k}_{\perp}} .
$$


Table 2: The Dziembowski wave function $\Psi\left(x, \mathbf{k}_{\perp}\right)$ and its FT $\hat{\Psi}(x, \mathbf{b})$

\begin{tabular}{|c|c|c|}
\hline$\lambda_{1} \lambda_{2}$ & $\Psi_{\lambda_{1} \lambda_{2}}\left(x, \mathbf{k}_{\perp}\right)$ & $\hat{\Psi}_{\lambda_{1} \lambda_{2}}(x, \mathbf{b})$ \\
\hline$\uparrow \uparrow$ & $-\left(a_{1 x}+a_{2 x}\right) k^{L} \hat{\phi}\left(x, k_{\perp}\right)$ & $i\left(a_{1 x}+a_{2 x}\right) b^{L} \xi(x, b) g(x) / 2$ \\
\hline$\uparrow \downarrow$ & $\left(a_{1 x} a_{2 x}-k_{\perp}^{2}\right) \hat{\phi}\left(x, k_{\perp}\right)$ & $\left(a_{1 x} a_{2 x}-g(x)\left(1-b^{2} g(x) / 4\right)\right) \xi(x, b)$ \\
\hline$\downarrow \uparrow$ & $-\left(a_{1 x} a_{2 x}-k_{\perp}^{2}\right) \hat{\phi}\left(x, k_{\perp}\right)$ & $-\left(a_{1 x} a_{2 x}-g(x)\left(1-b^{2} g(x) / 4\right)\right) \xi(x, b)$ \\
\hline$\downarrow \downarrow$ & $-\left(a_{1 x}+a_{2 x}\right) k^{R} \hat{\phi}\left(x, k_{\perp}\right)$ & $i\left(a_{1 x}+a_{2 x}\right) b^{R} \xi(x, b) g(x) / 2$ \\
\hline
\end{tabular}

The notation used in Tables 1 and 2 is:

$$
\begin{aligned}
a_{i x} & =x_{i} m_{\pi}+m, \quad \hat{\phi}\left(x, k_{\perp}\right)=\frac{\exp \left(-\frac{k_{\perp}^{2}+m^{2}}{8 \beta^{2} x(1-x)}\right)}{x(1-x)}, \quad k^{R(L)}=k_{x} \pm i k_{y}, \\
b^{R(L)} & =b_{x} \pm i b_{y}, \quad g(x)=8 \beta^{2} x(1-x), \quad \xi(x, b)=8 \pi \beta^{2} \exp \left(-\frac{b^{2} g(x)}{4}-\frac{m^{2}}{g(x)}\right) .
\end{aligned}
$$

Writing Eq. (罒) in terms of helicity components, we get

$$
\begin{aligned}
F_{\pi}\left(Q^{2}\right)= & \int \frac{d x d y}{\left(16 \pi^{3}\right)^{2}} \int \frac{b d b}{2 \pi} \hat{T}_{H}(x, y, \mathbf{b}, Q, t)\left[\hat{\Psi}_{\uparrow \downarrow}^{*}(y, \mathbf{b}) \hat{\Psi}_{\uparrow \downarrow}(x, \mathbf{b})+\hat{\Psi}_{\downarrow \uparrow}^{*}(y, \mathbf{b}) \hat{\Psi}_{\downarrow \uparrow}(x, \mathbf{b})\right. \\
& \left.-\left(\hat{\Psi}_{\uparrow \uparrow}^{*}(y, \mathbf{b}) \hat{\Psi}_{\uparrow \uparrow}(x, \mathbf{b})+\hat{\Psi}_{\downarrow \downarrow}^{*}(y, \mathbf{b}) \hat{\Psi}_{\downarrow \downarrow}(x, \mathbf{b})\right)\right] \exp (-S(x, y, b, Q, t)) .
\end{aligned}
$$

Before carrying out the calculation, we have to fix the parameters in the wave function. The constraint from $\pi \longrightarrow \mu \bar{\nu}$ decay requires that the wavefunction be normalized by the pion decay constant, $f_{\pi}$, through the relation

$$
\int \frac{d x d^{2} k_{\perp}}{16 \pi^{3}} \Psi\left(x, \mathbf{k}_{\perp}\right)=\frac{f_{\pi}}{2 \sqrt{n_{c}}},
$$

where $f_{\pi}=93 \mathrm{MeV}$ and $n_{c}=3$. In order for the theory to be physically sensible and self consistent, we make sure that the parameters, i.e., quark mass $m$ and harmonic oscillator parameter $\beta$, are chosen in such a way that while the equation (13) is satisfied, the calculated pion charge radius is approximately equal to its experimental value $\left(\left\langle r_{\pi}^{2}>_{\exp }^{1 / 2}=0.66 \mathrm{fm}[11]\right)\right.$.

The results of our numerical calculation are presented in Fig. 1, where the difference in $Q^{2} F_{\pi}\left(Q^{2}\right)$ with the inclusion and the exclusion of $h_{1}+h_{2}= \pm 1$ components as well as the Sudakov effects are shown. The curves (a) and (b) are for the naive helicity function and Dziembowski's form, respectively.

There are several aspects worth commenting here with respect to our calculation: 1) It is necessary to satisfy the physical constraints at low $Q^{2}$ and Eq. (13) if one is to study the transition from soft to hard QCD. With $m=330 \mathrm{MeV}, \beta=260$ (320) MeV for model 1 (2), we get $<r_{\pi}^{2}>^{1 / 2}=0.65(0.64) \mathrm{fm}$, respectively. It has been known that with a specific set of 

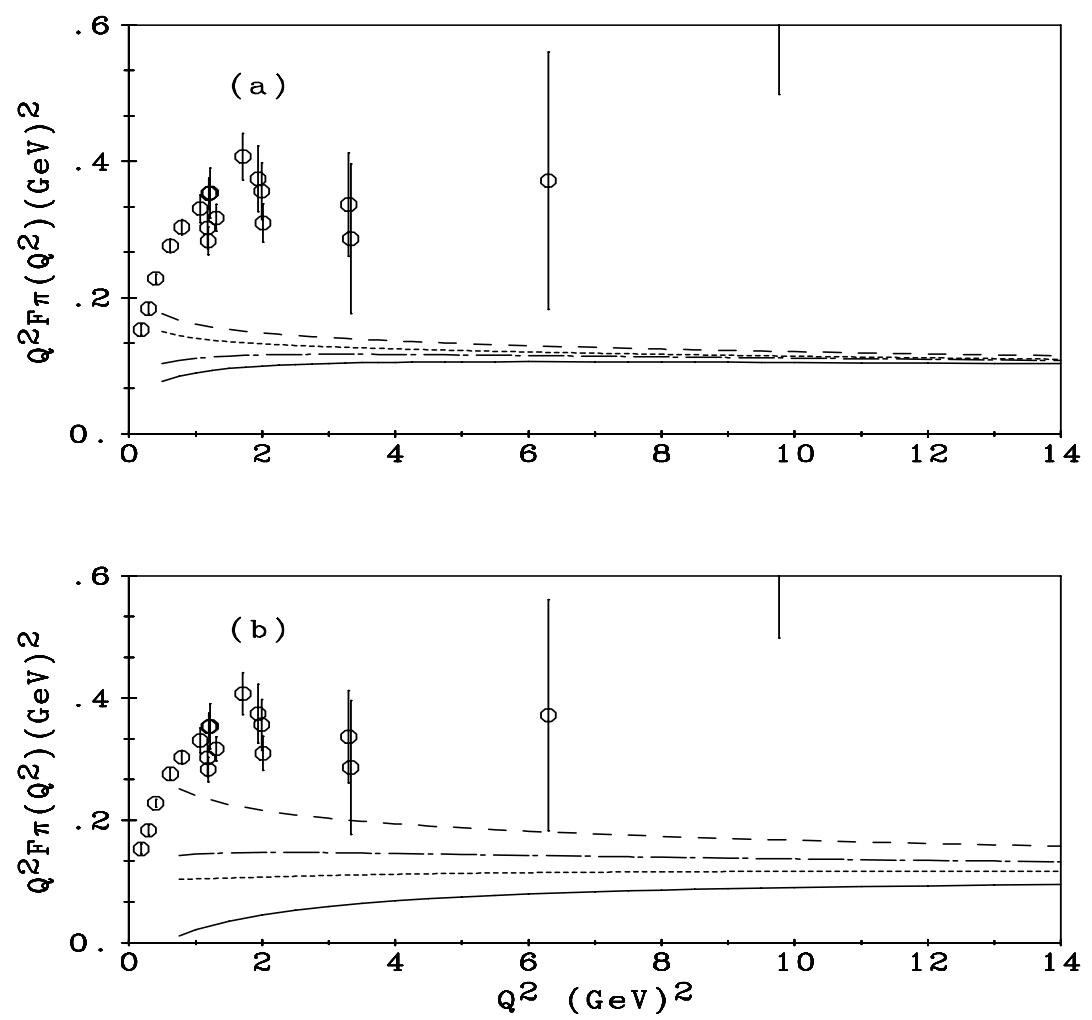

Figure 1: Pion form factors. The dashed and dotted lines are the results without or with $h_{1}+h_{2}= \pm 1$ components, respectively. The dash-dotted and solid lines are the corresponding results but with the Sudakov correction included. The naive helicity function Eq. (4) is used for Fig. (a) with $\beta=260 \mathrm{MeV}$, while for Fig. (b), Dziemboski's helicity function Eq. (5) is used with $\beta=320 \mathrm{MeV}$. The parameters: $m=330 \mathrm{MeV}$ and $\Lambda_{Q C D}=200 \mathrm{MeV}$ are the same for both $(a)$ and $(b)$. The experimental data are taken from 13 .

parameters, one can obtain a quark distribution amplitude (QDA) similar to that of CZ's from Dziembowski's wave function. However, using this set of parameters we have failed to produce any hard scattering form factors which are physically sensible below $Q^{2}=100$ $\mathrm{GeV}^{2}$. Other problems with this set of parameters have been pointed out in [12]. This again shows that the $k_{\perp}$ dependence is not as trivial as one might have naively speculated. 2) The numerical results with only $h_{1}+h_{2}=0$ components are similar to the standard hard scattering prediction using the asymptotic form of the QDA, which is likely due to the fact that the QDAs obtained from our wave functions are close in form to the asymptotic one. 3) Adding the $h_{1}+h_{2}= \pm 1$ contents suppresses the hard scattering significantly in the present experimentally accessible energy region regardless the Sudakov correction. This suppression shows up for both helicity model wave functions but much stronger with Dziembowski's form as can be seen from Fig. 1(b). 
4) The contribution from $h_{1}+h_{2}= \pm 1$ becomes less important in large $Q^{2}$. For model 1 , this contribution vanishes beyond $Q^{2}=20 \mathrm{GeV}^{2}$. For model 2, the contribution is less than $5 \%$ at about $100 \mathrm{GeV}^{2}$ while it generates a suppression of factor two for the form factor at $2 \mathrm{GeV}^{2}$. As $Q^{2}$ goes to infinity, we recover the asymptotic prediction for both models. Therefore the conventional hard scattering theory remains intact only when $Q^{2} \rightarrow$ $\infty$. 5) Finally, in our analysis we neglect the quark propagator in the $T_{H}$ for our focus is on the helicity structure itself. This is justified by our result that the suppression from the unconventional helicity components is nearly 10 time larger than the effect from inclusion of the quark propagator at $2 \mathrm{GeV}^{2}$ for the model wavefunction 2 .

In conclusion, we have re-investigated the hard scattering pion form factor using the modified scattering amplitude $T_{H}$ with transverse momentum included. Special attention has been paid to the unconventional helicity components which are customarily neglected. We have shown that inclusion of these components suppresses the hard scattering at moderate $Q^{2}$ due to the fact that $T_{H}$ associated with $h_{1}+h_{2}= \pm 1$ bears an opposite sign in comparison with $h_{1}+h_{2}=0$ portions. Although this analysis is carried out using two particular models, the general consistency of the picture is certainly a further indication that the hard scattering mechanism is not the dominant one in the moderate $Q^{2}$ region, where experimental results are available. Therefore, there must be a quite sizable soft contribution as we have pointed out in [5, 6].

Acknowledgement: This work is supported in part by NSF grant PHY-9023586 and in part by DOE Grant DOE-FG02-93ER-40762.

\section{References}

[1] G.P. Lepage and S.J. Brodsky, Phys. Rev. D22, 2157 (1980), for technical details, see also G.P. Lepage, S.J. Brodsky, T. Huang and P.B. Mackenzie, Banff Summer Institute, Particles and Fields 2, eds. A.Z. Capri and A.N. Kamal (1983).

[2] N. Isgur and C.H. Liewellyn Smith, Phys. Rev. Lett.52, 1080 (1984); Phys. Lett. B217, 535 (1989); Nucl. Phys. B317, 526 (1989).

[3] V.L. Chernyak and A.R. Zhitnitsky, Nucl. Phys. B201, 492 (1982); B214, 547(E) (1983).

[4] H.N. Li and G. Sterman, Nucl. Phys. B381, 129 (1992); J. Botts and G. Sterman, Nucl. Phys. B325, 62 (1989); H.N. Li, Phys. Rev. D48, 4243 (1993).

[5] L.S. Kisslinger and S.W. Wang, Nucl. Phys. B399, 63 (1993); O.C. Jacob and L.S. Kisslinger, Phys. Lett. B243, 323 (1990).

[6] L.S. Kisslinger and S.W. Wang, Carnegie-Mellon preprint (1994), hep-ph/9403261.

[7] R. Jacob and P. Kroll, Phys. Lett. B315, 463 (1993). 
[8] A. Kondratyuk and M.V. Terentev, Yad. Fiz. 31, 1087 (1980) [Sov. J. Nucl. Phys. 31, 561 (1980)]; I.G. Aznauryan, A.S. Bagdasaryan, and N.L. Ter-lsaakyan, Yad. Fiz.36, 1278 (1982)[Sov. J. Nucl. Phys. 36, 743 (1982)]; Phys. Lett. B112, 393 (1982); B.D. Keister and W.N. Polyzou, Advances in Nuclear Physics, Vol. 20, eds. J.W. Negele and E. Vogt. Plenum, New York (1991).

[9] H.J. Melosh, Phys. Rev. D9, 1095 (1974).

[10] Z. Dziembowski, Phys. Rev. D37, 778 (1988).

[11] E.B. Dally et al., Phys. Rev. Lett. 48, 375 (1982).

[12] C.-R. Ji, P.L. Chung and S.R. Cotanch, Phys. Rev. D45, 4214 (1992).

[13] L.J. Bebek et al., Phys. Rev. D9, 1229 (1974); D13, 25 (1976); D17, 1693 (1978); C.N. Brown et al., Phys. Rev. D8, 92 (1973). 